\title{
Dirac and Majorana Spinors on Non-Orientable Riemann Surfaces ${ }^{\star}$
}

\author{
Benjamin Grinstein and Ryan Rohm $\star \star$ \\ Department of Physics, California Institute of Technology, Pasadena, CA 91125, USA
}

\begin{abstract}
It is well known that (Weyl) spinors cannot be consistently defined on nonorientable manifolds. We prove that Dirac spinors can be defined on nonorientable Riemann surfaces. It is also shown that Majorana spinors cannot be defined consistently on closed nonorientable Riemann surfaces with odd Euler number, but can be consistently defined in all other cases.
\end{abstract}

The recent surge of interest in string theories was triggered by the demonstration of the one loop cancellation of anomalies in the type I SO(32) superstring [1]. This calculation was carried out using the canonical quantization procedure. Green and Schwarz had already realized that similar infinity cancellations must be operative at tree level, but they could not prove the vanishing of the dilaton tadpole at this level since it was not clear how the canonical quantization procedure could be used to obtain this result. The alternative path integral quantization method of Polyakov can be used for this purpose [2]. In fact, it was recently used to show the vanishing of the dilaton tadpole for the $S O(8192)$ type I bosonic string theory [3].

The path integral quantization procedure for field theories is notorious for its suitability in discussing global obstructions to the consistent formulation of the theory. For example, Witten has shown that a class of gauge theories with spinors is inconsistent [4], but the corresponding anomaly is much more difficult to understand if canonical quantization methods are used [5]. Polyakov's formulation of the super-string can then be expected to shed new light on possible global inconsistencies of superstring theories.

The partition function in Polyakov's theory is a sum over two-dimensional field theories defined on different Riemann surfaces. For the type I superstring, it includes both open and closed, oriented and nonoriented surfaces. The formulation is in Euclidean space and the action is just the integral of the supergravity

* Work supported in part by the U.S. Department of Energy under contract DEAC 03-81-ER40050

$\star \star$ Weingart Research Fellow 
Lagrangian discovered by Brink et al. [6], and by Deser and Zumino [7]. All the spinors are taken to satisfy a Majorana condition, and the proofs of the supersymmetry of the action given in [6] and [7] rely on this fact. In this work, we will prove that Majorana spinors cannot be consistently defined on closed unoriented manifolds with odd Euler characteristics. Therefore, Polyakov's procedure is inconsistent as it stands.

It is well known that a spin structure cannot be defined on unoriented spaces. This is easy to understand since it is just the statement that closed paths exist on which a left-handed Weyl spinor can be parallel transported to come back to the starting point as a right-handed spinor. It is not clear a priori whether Dirac spinors, which contain both handedness, can be consistently defined on an unoriented space. In fact, we will see that this is the case when the space is a Riemann surface. However, the definition is incompatible with the reality condition defining Majorana spinors in the case of odd Euler number.

We start by focusing on some simple cases. Let $U=\left\{U_{\alpha}\right\}$ be a good cover of the Riemann surface $M$. Recall that in constructing a spin bundle, one needs first a frame bundle. This is specified by a set of transition functions $R_{\alpha \beta} \in O(2)$ defined on the overlaps $U_{\alpha \beta} \in U_{\alpha} \cap U_{\beta}$. On the overlaps the frames $e_{\alpha}^{a}(a=1,2)$ are related by

$$
e_{\alpha}^{a}=\left(R_{\alpha \beta}\right)_{b}^{a} e_{\beta}^{b} .
$$

To construct the spin bundle, one then needs to lift these transition functions to elements $L_{\alpha \beta}$ of the double covering of $O(2)$, namely Pin(2) [8]. On the overlaps, spinors $\psi_{\alpha}^{i}(i=1,2)$ are related by

$$
\psi_{\alpha}^{i}=\left(L_{\alpha \beta}\right)_{j}^{i} \psi_{\beta}^{i} .
$$

Now consider the specific case of $M$ being the projective plane $P_{2}$, viewed as the disk with antipodal points of its boundary identified. For simplicity, choose a cover with double overlaps covering the entire boundary of the disk and additional double overlaps entirely in the interior of the disk (see Fig. 1). The transition functions $R_{\alpha \beta}$ can be chosen to be trivial in the overlaps interior to the disk. We can view this situation by putting an everywhere parallel frame in the interior of the disk. The nontrivial transition functions then lie on the boundary of the disk and can be computed by "moving" the frame through the boundary and finding out their orientation as they come in through the antipodal point. The situation is depicted in Fig. 2. Parametrizing the boundary of the disk by an angular variable $\phi \in[0,2 \pi)$, with $\phi=0$ on the south pole " $A$ " of Fig. 2 , one has

$$
R(\phi)=P e^{i T 2 \phi}=P\left(\begin{array}{rr}
\cos 2 \phi & -\sin 2 \phi \\
\sin 2 \phi & \cos 2 \phi
\end{array}\right),
$$

where

$$
P=\left(\begin{array}{rr}
1 & 0 \\
0 & -1
\end{array}\right) \in O(2)
$$

is the "parity" matrix and

$$
T=\left(\begin{array}{cc}
0 & i \\
-i & 0
\end{array}\right) \in \text { Lie algebra of } S O(2)
$$




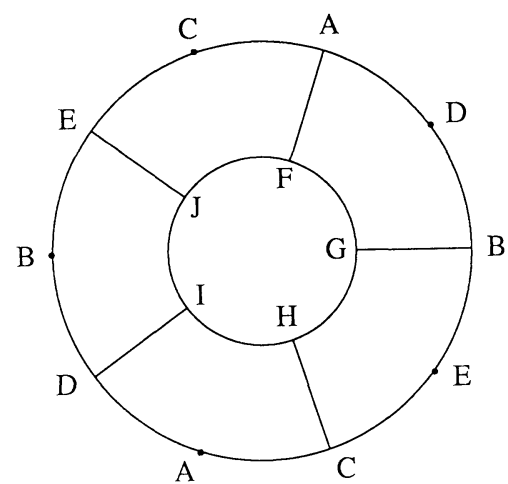

Fig. 1. $P_{2}$ as the disk with antipodal points of its boundary identified. Drawn in is a cover, with triple overlaps labeled by letters $A, \ldots, J$

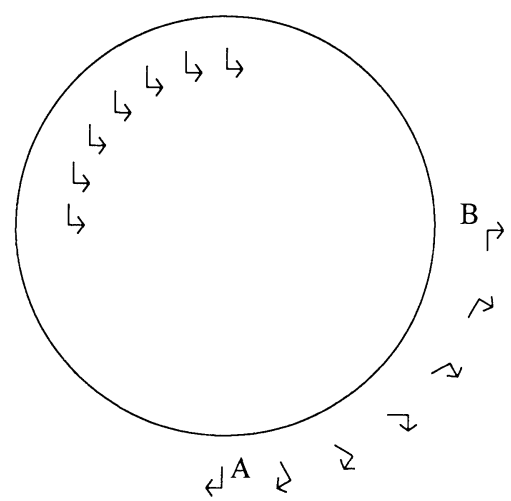

Fig. 2. The transition function for the frame on the overlaps $\overline{A B}$. Note that the frame rotates by $\pi$ as we traverse an angle $\pi / 2$

is the hermitian generator of rotations. Notice that frames rotate by $4 \pi$ as we traverse the boundary of the disk. Now, in defining the corresponding transition function $L(\phi)$ for spinors $\psi$, we must require that $\psi^{\dagger} \psi$ and $\psi^{\dagger} \gamma^{a} \psi$ transform as scalar and vector, respectively. Here and throughout $\gamma^{a}=\sigma^{a}$, the Pauli spin matrices. This imposes the following conditions

$$
\begin{aligned}
L^{\dagger}(\phi) L(\phi) & =1, \\
L^{\dagger}(\phi) \gamma^{a} L(\phi) & =R(\phi)_{b}^{a} \gamma^{b},
\end{aligned}
$$

which are solved by

$$
L(\phi)=e^{i \omega} \gamma^{1} e^{i \gamma^{3} \phi} .
$$

The phase $w$ can be further restricted by requiring that $\psi^{c \dagger} \gamma^{3} \psi$ be a scalar, where $\psi^{c}$ is the charge conjugate of $\psi$. The extra freedom arises because the previous two requirements were consistent with the presence of a background $U(1)$ gauge field; one can eliminate this arbitrariness by requiring the charged fermion bilinear 
$\psi^{c \dagger} \gamma^{3} \psi$ to transform as a scalar. We will come back to this point later, but for the moment we will keep $w$ unrestricted. Our solution for $L(\phi)$ states that

$$
\psi(\pi)=e^{i w} \gamma^{1} \psi(0)
$$

and that

$$
\psi(2 \pi)=\psi(0)=e^{i w} \gamma^{1} e^{i \gamma^{3} \pi} \psi(\pi) .
$$

Here $\psi(\phi)$ refers to the spinor on the patch $U_{\alpha}$ that extends to the interior of the disk and contains that point $\phi$ of the boundary. Combining these expressions gives the condition

$$
e^{2 i w}=-1
$$

Thus Dirac spinors can be defined on $P_{2}$ provided $e^{i w}= \pm i$. Majorana spinors satisfy the additional constraint

$$
\psi^{c}=\psi
$$

Here $\psi^{c}$ is the charge conjugate spinor. It is defined to be

$$
\psi^{c}=e^{i \alpha} C \psi,
$$

where $\alpha$ is an arbitrary phase and the charge conjugation matrix satisfies ${ }^{1}$

$$
C^{-1} \gamma^{\alpha} C=\gamma^{a *}
$$

and

$$
C^{*} C=1
$$

With our conventions $C=\gamma^{1}$.

If the spinor $\psi$ is Majorana, it must satisfy

$$
\psi(\pi)=e^{i w} \gamma^{1} \psi(0)
$$

and its $C$ conjugate relation

$$
\psi(\pi)=e^{-i w} \gamma^{1} \psi(0)
$$

Thus one needs

$$
e^{2 i w}=1
$$

which is impossible in light of Eq. (8). There are no Majorana spinors on $P_{2}$. One may be skeptical about this derivation since the phases $w$ and $\alpha$ could have been chosen to vary smoothly along the boundary. It is a trivial exercise to show that this added freedom does not modify our conclusion. The fact that the cover is somewhat special is also superfluous: we will later prove that the problem exists for any cover and any continuous local choice of phases $w$ and $\alpha$. The exercise above does indeed contain all the essential ingredients of the generic proof.

\footnotetext{
${ }^{1}$ If one requires that $C^{-1} \gamma^{a} C=-\gamma^{a *}$, then it is not possible to satisfy $C^{*} C=1$ and a Majorana condition is inconsistent. The condition in Eq. (11) still implies $C^{-1} \sum^{a b} C=\sum^{a b *}$, where $\sum^{a b}=-\frac{i}{4}\left[\gamma^{a}, \gamma^{b}\right]$ are the hermitian generators of rotations $\left(\sum^{12}=\frac{1}{2} \gamma^{3}\right)$
} 


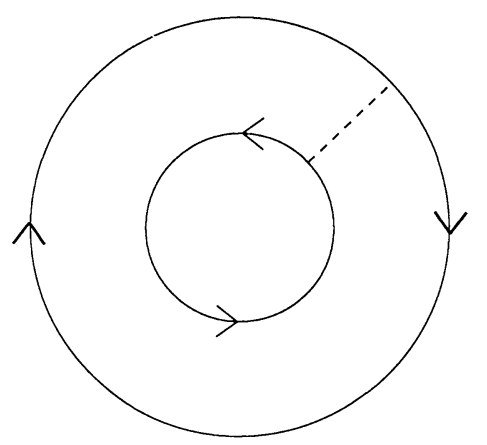

Fig. 3. The Klein bottle can constructed from the annulus by identifying antipodal points on each boundary. The spinor transition function can be nontrivial across the dotted line

In the next example, we take $M$ to be the Klein bottle $K_{2}$, which can be obtained from the above construction of $P_{2}$ by removing a small open disk from its interior and identifying antipodal points of the new boundary (i.e., the annulus with antipodal identification of points in both boundaries, as in Fig. 3). By retracing the argument for $P_{2}$, it is obvious that one can define Dirac spinors on $K_{2}$. The fact that one can have Majorana spinors depends on a simple observation. Consider a line $\ell$ joining the inner and outer boundaries of the annulus, as represented by the dotted line in Fig. 3. We are still considering an everywhere parallel frame on the annulus, so the transition function for spinors on $\ell$ is just $L= \pm 1$ (a phase in general, but \pm 1 if we want to consider Majorana spinors, for $\alpha=0$ ). If we parametrize the transition functions on the boundaries of the annulus by phases $\phi_{1}$ and $\phi_{2}$ as before, with $\phi_{i}=0$ corresponding to the points where the boundaries meet the line $\ell$, then the transition functions are (if $L=-1$ across $\ell$ )

$$
L\left(\phi_{i}\right)=\gamma^{1} e^{i \gamma^{3} \phi_{i}} \text { for } 0 \leqq \phi_{i}<\pi
$$

and

$$
L\left(\phi_{i}\right)=-\gamma^{1} e^{i \gamma^{3} \phi_{i}} \text { for } \pi \leqq \phi_{i}<2 \pi,
$$

corresponding to the case $e^{2 i w}=1$. There are Majorana spinors on $K_{2}$. Note that this procedure could not have worked on $P_{2}$, since the line $\ell$ cannot end in the interior of the disk (for then the transition function on some overlap is discontinuous) and we gain nothing by having it end somewhere else on the boundary (for then $e^{2 i w}=-1$ still).

It is now easy to understand the general case. A disk with $1-\chi$ holes with antipodal identification on all boundaries is a closed unoriented Riemann surface with Euler number $\chi$ [9]. Again, we put an everywhere parallel frame on this disk with holes. If $\chi$ is even, we can have $1-\chi / 2$ lines joining pairs of cross-caps on which we can choose the spinor transition function to be nontrivial and Majorana spinors can be defined. If $\chi$ is odd, this is not possible. It is also easy to see what happens for open unoriented surfaces. One typically imposes a boundary condition for the spinors, but the condition being local, we can draw a line $\ell$ from a 
cross cap and have it end at the boundary without introducing discontinuous transition functions. There is therefore no obstruction to defining Majorana spinors on open unoriented surfaces. ${ }^{2}$

To make a proof out of the preceding argument, we need only a good bookkeeping device to keep track of the freedom in the choices of phases. The natural tool is Čech cohomology. An excellent introduction can be found in [10]. Choose a good cover $\mathscr{U}=\left\{U_{\alpha}\right\}$ of the closed Riemann surface $M$. A frame bundle is specified by the transition functions $R_{\alpha \beta} \in O(2)$ on the overlaps $U_{\alpha \beta}=U_{\alpha} \cap U_{\beta}$; here $U_{\alpha \beta}$ is to be thought of as a two-chain in $C_{2}(\mathscr{U})$. Consistency of Eq. (1) requires $R_{\alpha \beta}$ to satisfy the cocycle condition on triple overlaps $U_{\alpha \beta \gamma} \in C_{3}(\mathscr{U})$ :

$$
\delta\{R\}_{\alpha \beta \gamma}=R_{\alpha \beta} R_{\beta \gamma} R_{\gamma \alpha}=1 .
$$

As in Eq. (3), $R_{\alpha \beta}$ can always be written as

$$
R_{\alpha \beta}=P^{p_{\alpha \beta}} e^{i T \phi_{\alpha \beta}}
$$

with

$$
\phi_{\beta \alpha}=-(-1)^{p_{\alpha \beta}} \phi_{\alpha \beta},
$$

and the cocycle condition becomes

$$
(-1)^{p_{\beta \gamma}+p_{\gamma \alpha}} \phi_{\alpha \beta}+(-1)^{p_{\gamma \alpha}} \phi_{\beta \gamma}+\phi_{\gamma \alpha}=2 \pi n_{\alpha \beta \gamma}
$$

for some integer $n_{\alpha \beta \gamma}$. A bundle of Dirac spinors can be constructed if the transition functions $L_{\alpha \beta} \in \operatorname{Pin}(2)$ can be chosen to satisfy a cocycle condition. Let

$$
\overline{\mathrm{w}}_{\alpha \beta \gamma}=\delta\{L\}_{\alpha \beta \gamma} \text {. }
$$

We will begin by showing that there are no Majorana spinors for odd Euler number. We want to prove that the $L_{\alpha \beta}$ can be chosen so that $\overline{\mathrm{w}}_{\alpha \beta \gamma}=1$. We have already seen that the condition that $\psi^{\dagger} \psi$ and $\psi^{\dagger} \gamma^{\alpha} \psi$ transform as scalar and vector, respectively, fixes the form of $L_{\alpha \beta}$ to be

$$
L_{\alpha \beta}=e^{i w_{\alpha \beta}}\left(\gamma^{1}\right)^{p_{\alpha \beta}} e^{i \frac{\gamma^{3}}{2} \phi_{\alpha \beta}} .
$$

Using Eqs. (16) and (17), one obtains

$$
\overline{\mathrm{W}}_{\alpha \beta \gamma}=e^{i \delta\{w\}_{\alpha \beta \gamma}}(-1)^{n_{\alpha \beta \gamma}},
$$

the coboundary operator on phases $\left(w_{\alpha \beta}=-w_{\beta \alpha}\right)$ is

$$
\delta\{w\}_{\alpha \beta \gamma}=w_{\alpha \beta}+w_{\beta \gamma}+w_{\gamma \alpha}
$$

and we have used

$$
\delta\{p\}_{\alpha \beta \gamma}=0 \bmod 2 .
$$

It is easy to check $^{3}$ that $\mathrm{w}_{\alpha \beta \gamma}=(-1)^{n_{\alpha \beta \gamma}}$ is a two-cocycle: furthermore, it is a representative of the second Stiefel-Whitney class $\mathrm{w}_{2} \in H^{2}\left(M, \mathbf{Z}_{2}\right)$ [11]. Because

\footnotetext{
${ }^{2}$ Note, however, that this choice picks out the boundary conditions satisfied by the spinor around this boundary: on an open nonoriented Riemann surface, the number of boundaries around which the spin structure is odd will be odd if $\chi$ is odd and even if $\chi$ is even

${ }^{3}$ With the aid of Eq. (15) and (16) one can show that $\delta\{n\}_{\alpha \beta \gamma \delta}$ is an integral multiple of $4 \pi$
} 
$d=2, \mathrm{w}_{2}$ is the $\bmod 2$ Euler class $(\chi \bmod 2$ times the nontrivial two-cocycle). For Majorana spinors, one must choose the phases $w_{\alpha \beta}$ to be multiples of $\pi$, so that the two-boundary $e^{i \delta\{\boldsymbol{w}\} \alpha \beta \gamma}$ takes values in $\mathbf{Z}_{2}$. One can then arrange [11] for $\overline{\mathrm{w}}_{\alpha \beta \gamma}=1$ only if $\mathrm{w}_{2}$ is a trivial cohomology class, that is, if $\chi=0 \bmod 2$. Conversely, if the phases $w_{\alpha \beta}$ are taken to be multiples of $\pi$, then one can never arrange to have $\overline{\mathrm{w}}_{\alpha \beta \gamma}=1$ in all the overlaps if $\chi=1 \bmod 2$.

If we allow for a phase freedom in the definition of Majorana spinors, we still get the same result. The general Majorana condition is

$$
\psi_{\alpha}=e^{i c_{\alpha}} C \psi_{\alpha}^{*} .
$$

From Eq. (2) one learns that a Majorana spinor bundle satisfies

$$
L_{\alpha \beta}=e^{i \delta\left\{c c_{\alpha \beta}\right.} C L_{\alpha \beta}^{*} C^{-1}
$$

or simply, that

$$
e^{2 i w_{\alpha \beta}}=e^{i \delta\{c\}_{\alpha \beta}} .
$$

Therefore, $w_{\alpha \beta}$ must be a multiple of $\pi$ modulo an irrelevant 1-coboundary.

We still have to show that there are Dirac spinors on these manifolds. This would be obvious if the phases $w_{\alpha \beta}$ could be chosen arbitrarily. One additional and last constraint arises from requiring that $\psi^{\dagger} \gamma^{3} \psi^{c}$ transforms like a scalar. This gives

$$
C^{T} L_{\alpha \beta}^{T} C^{\dagger} \gamma^{3} L_{\alpha \beta} e^{-i \delta\left\{c c_{\alpha \beta}\right.}=\gamma^{3}
$$

or

$$
e^{2 i w_{\alpha \beta}-i \delta\{c\}_{\alpha \beta}}(-1)^{p_{\alpha \beta}}=1
$$

Thus

$$
w_{\alpha \beta}=\delta\{c\}_{\alpha \beta}+\frac{\pi}{4}\left(1-(-1)^{p_{\alpha \beta}}\right)+m_{\alpha \beta} \pi
$$

with $m_{\alpha \beta}$ an integer, and finally

$$
\overline{\mathrm{W}}_{\alpha \beta \gamma}=(-1)^{\frac{1}{2} \delta\{p\}_{\alpha \beta \gamma}}(-1)^{\delta\{m\}_{\alpha \beta \gamma}} \mathrm{W}_{\alpha \beta \gamma} .
$$

The first factor in Eq. (28) has an interesting derivation. The factor of $1 / 2$ in the exponent is all that prevents it from being trivial; $\{p\}$ represents a one-dimensional cohomology class with $\mathbf{Z}_{2}$ coefficients, calculated from the occurrences of the orientation-changing matrix $P$ in the transition functions. $\{p\}$ is nontrivial if and only if the manifold is nonorientable, and it represents the first Stiefel-Whitney class $\mathrm{w}_{1} \in H^{1}\left(M, \mathbf{Z}_{2}\right)$; trivially, then, $\delta\{p\}$ is zero as a $\mathbf{Z}_{2}$ cochain. The factor of $1 / 2$ in Eq. (28) then corresponds to a cohomology operation known as a Steenrod square $[12,13]$. Diagram chasers may wish to peruse the following figure:

$$
\begin{aligned}
& \left(S q^{1}\left(\mathrm{w}_{1}\right)\right) \leftarrow \delta\left(\mathrm{w}_{1}\right)_{\mathbf{z}_{4}} \\
& 0 \rightarrow C^{(2)}\left(M, \mathbf{Z}_{2}\right) \rightarrow C^{(2)}\left(M, \mathbf{Z}_{4}\right) \rightarrow C^{(2)}\left(M, \mathbf{Z}_{2}\right) \rightarrow 0 \\
& \uparrow_{\delta} \quad \uparrow \uparrow_{\delta} \quad \uparrow_{\delta} \\
& 0 \rightarrow C^{(1)}\left(M, \mathbf{Z}_{1}\right) \rightarrow C^{(1)}\left(M, \mathbf{Z}_{4}\right) \rightarrow C^{(1)}\left(M, \mathbf{Z}_{2}\right) \rightarrow 0 \\
& \left(\mathrm{w}_{1}\right)_{\mathbf{z}_{4}} \leftarrow\left(\mathrm{w}_{1}\right)
\end{aligned}
$$


From a cochain representing a cohomology class in $H^{1}\left(M, \mathbf{Z}_{2}\right)$ (here, $\left.\{p\}\right)$ one forms a cochain with $\mathbf{Z}_{4}$ coefficients by forgetting that the coefficients are $\mathbf{Z}_{2}$. This cochain may not be closed, but we know that $\delta\{p\}$, evaluated with $\mathbf{Z}_{4}$ coefficients, has coefficients which are multiples of two, from the fact that $\delta\{p\}$ is zero as a $\mathbf{Z}_{2}$ cocycle. We can then divide the coefficients by two to get a two-cochain with $\mathbf{Z}_{2}$ coefficients, and this whole operation is written symbolically as $S q^{1} \mathrm{w}_{1}$.

From the defining relations of Steenrod squares [13] we have the formula

$$
S q^{1}\left(\mathrm{w}_{1}\right)=\mathrm{w}_{1} \cup \mathrm{w}_{1} .
$$

This can be computed using homology cycles Poincaré dual to $\mathrm{w}_{1}$ and taking their intersection. Representing a closed nonorientable surface by a sphere with $2-\chi$ crosscaps, the cycle dual to $\mathrm{w}_{1}$ is a sum of loops through the crosscaps, each of which has self-intersection equal to a point. The intersection is then a set of $2-\chi$ points, which is trivial if $\chi$ is even and nontrivial if $\chi$ is odd.

This gives us

$$
S q^{1}\left(\mathrm{w}_{1}(M)\right)=\mathrm{w}_{2}(M)
$$

for nonorientable Riemann surfaces; if we then take cohomology classes in Eq. (28), we have $[\overline{\mathrm{w}}]=\left[S q^{1}\left(\mathrm{w}_{1}\right)\right]+\left[\mathrm{w}_{2}\right]=2\left[\mathrm{w}_{2}\right]=0$. Thus there is no obstruction to defining Dirac spinors.

Given that there are no Majorana spinors on odd Euler surfaces, can we still have a path integral formulation of the type I superstring? One possibility is to define the theory in Minkowski space and to perform the Wick rotation to Euclidean space only after the local supersymmetry has been used up to fix the gauge. It is not clear whether the non-existence of Majorana spinors would introduce technical obstructions in performing the Wick rotation. Since the NSRsuperstring action contains an even number of Majorana spinors, a second approach consists of finding a similar action with half as many Dirac spinors which still exhibits the local supersymmetry. For example, the gauge fixed action in [6] and [7] when viewed as a functional of five complex scalars and Dirac spinors still presents a supersymmetry. This is generated by a conformal killing spinor which need not be Majorana. It is hard to see how one could extend this procedure to the full action. In particular, one would have to pair the two Majorana components of the gravitino into a Dirac spinor.

We have proven that Dirac spinors can be defined on all Riemann surfaces, and that Majorana spinors are inconsistent only for closed surfaces with odd Euler number. It would be interesting to know if Majorana spinors can be consistently defined on unoriented manifolds of higher dimensions and on unoriented twodimensional spaces with Minkowski metric. Also of interest is the classification of spin structures and of Majorana spin structures on Riemann surfaces. With Preskill we are presently pursuing these and related questions. 


\section{References}

1. Green, M.B., Schwarz, J.H.: Infinity cancellations in $S O(32)$ superstring theory. Phys. Lett. $151 \mathrm{~B}, 21$ (1985); Anomaly cancellations in supersymmetric $D=10$ gauge theory and superstring theory. idem 149 B, 117 (1984); The hexagon gauge anomaly in type 1 superstring theory. Nucl. Phys. B 255, 93 (1985)

2. Polyakov, A.M.: Quantum geometry of bosonic strings. Phys. Lett. 103B, 207 (1981); Quantum geometry of fermionic strings. idem 103B, 211 (1981)

3. Grinstein, B., Wise, M.: Vacuum energy and dilaton tadpole for the unoriented closed bosonic string. Phys. Rev. D 35, 655 (1987);

Douglas, M., Grinstein, B.: Phys. Lett. 183B, 52 (1987)

4. Witten, E.: An SU(2) anomaly. Phys. Lett. 117B, 324 (1982)

5. Sonoda, H.: The Wess-Zumino term and the Hamiltonian formulation for anomalies. Phys. Lett. 156B, 220 (1985)

6. Brink, L., DiVecchia, P., Howe, P.: A locally supersymmetric and reparametrization invariant action for the spinning string. Phys. Lett. 65B, 471 (1976)

7. Deser, S., Zumino, B.: A complete action for the spinning string. Phys. Lett. 65B, 369 (1976)

8. Atiyah, M.F., Bott, R., Shapiro, A.: Topology 3, 3 (1964)

9. Armstrong, M.A.: Basic topology. Berlin, Heidelberg, New York: Springer 1983

10. Alvarez, O.: Topological quantization and cohomology. Commun. Math. Phys. 100, 279 (1985)

11. Alvarez-Gaumé, L., Nelson, P.: CERN preprint CERN-TH-4615/86. December 1986

12. Milnor, J.W., Stasheff, J.D.: Characteristic classes. Princeton, NJ: Princeton University Press 1974

13. Spanier, E.H.: Algebraic topology. Berlin, Heidelberg, New York: Springer 1966

Communicated by L. Alvarez-Gaumé

Received March 13, 1987 
